\title{
Spondyloepimetaphyseal dysplasia with joint laxity (SEMDJL)
}

\author{
P Beighton
}

MRC Research Unit for Medical Genetics, Department of Human Genetics, Medical School, University of Cape Town, Observatory 7925, South Africa $P$ Beighton
Spondyloepimetaphyseal dysplasia with joint laxity (SEMDJL) (MIM 271640) is a rare autosomal recessive skeletal dysplasia which is characterised by dwarfism, articular hypermobility, and progressive intractable spinal malalignment. A typical facies, cleft palate, and a propensity to joint dislocation and subluxation are additional features. The condition is potentially lethal owing to cardiorespiratory compromise and complications consequent upon spinal cord compression; survival to adulthood is unusual. The condition is predominantly found in the Afrikaans speaking population of South Africa, where 20 affected children have been identified. SEMDJL has received the asterisk of proven syndromic identity in Mendelian inheritance in man $^{1}$ and the disorder is listed in the International classification of the chondrodysplasias. ${ }^{2}$

\section{Historical introduction}

In 1979, during a review of undiagnosed cases in the Skeletal Dysplasia Registry of the Department of Human Genetics, Medical School, University of Cape Town, seven children with similar clinical and radiographic features were recognised. An account of this disorder was subsequently published by Beighton and Kozlowski ${ }^{3}$ under the title "Spondylo-epi-metaphyseal dysplasia with joint laxity and progressive scoliosis". Thereafter further affected children were encountered in South Africa during diagnostic screening at special facilities for the physically handicapped and at outreach genetic clinics. The manifestations of 18 South African children with the condition were reviewed, ${ }^{4}$ and a detailed account of their radiographic features followed. ${ }^{5}$ Confirmation of the autosomal recessive mode of inheritance of the disorder was obtained at this stage. ${ }^{6}$ Comprehensive genealogical investigations involving archival re-

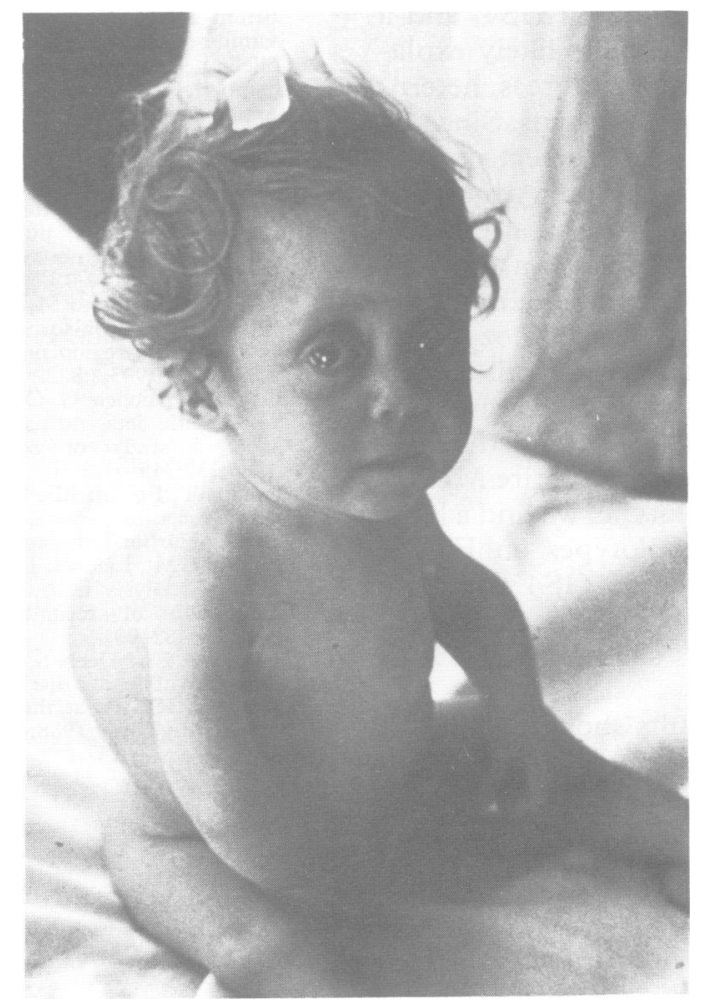

Figure 1 The phenotype in infancy. This dwarfed child has obvious dorsal kyphoscoliosis. The face is oval with protuberant eyes and a long upper lip. (From Beighton et al. Clin Genet 1984; 26: 308-17.)

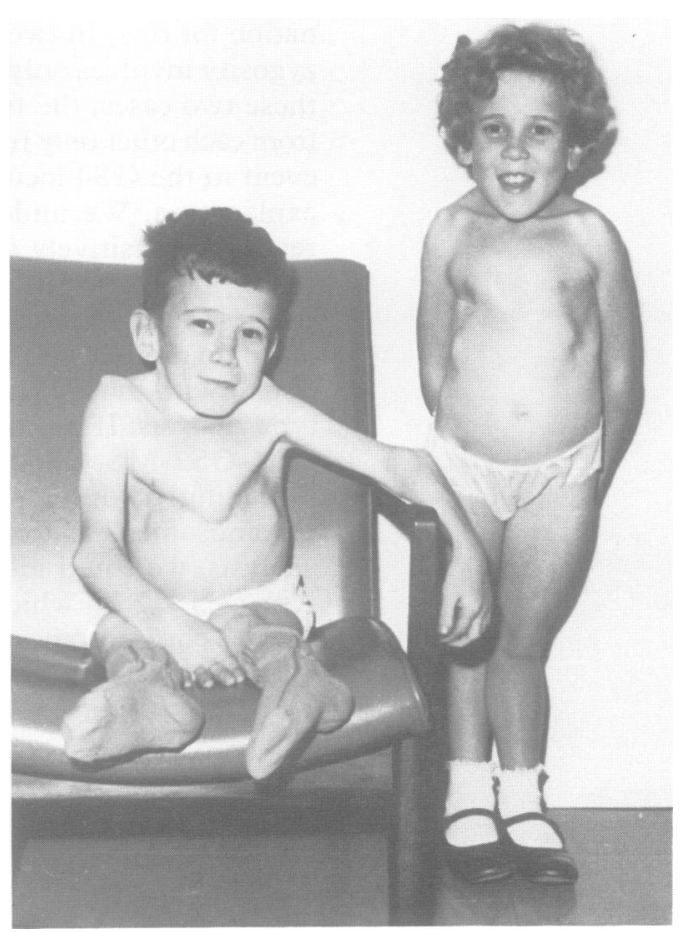

Figure $2 A$ brother and sister with SEMDYL. Both have dwarfism and thoracic asymmetry and the boy is paraplegic owing to spinal cord compression. Bilateral radial head dislocation is evident in the boy and his sister has genu valgus. 


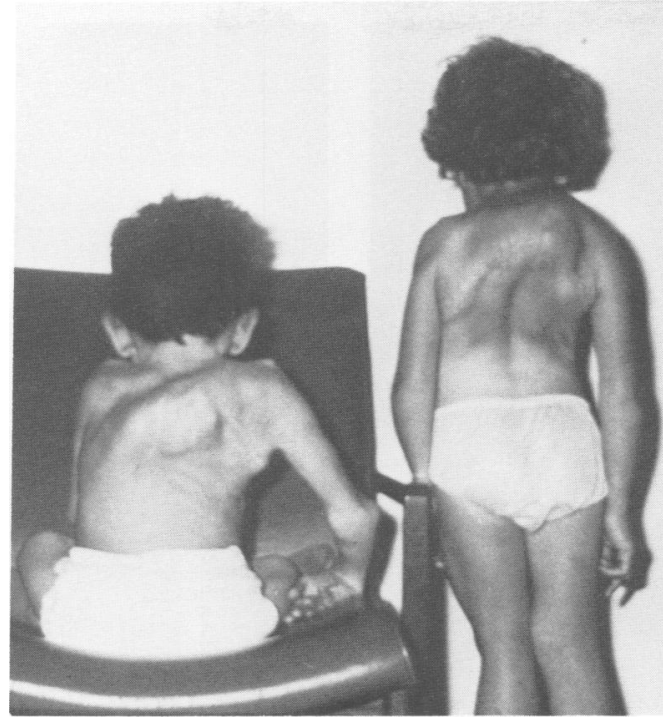

Figure 3 Sibs with SEMDFL. Severe kyphoscoliosis has proved refractory to operative treatment.

sources led to the identification of two females who had links, 12 generations removed, with eight of the affected families. ${ }^{7}$ Investigations of the basic biomolecular defect are currently under way.

\section{Clinical manifestations}

SEMDJL presents at birth as dwarfism with articular abnormalities which are the consequences of joint laxity, notably consistent dislocation of the radial heads and inconsistent talipes equinovarus. At this stage the thorax is usually asymmetrical and the spine may be malaligned.

The range of joint movements is excessive, although the elbows show paradoxical limitation of full extension and rotation, owing to radial head dislocation. The hypermobility is especially evident in the digits, which can assume bizarre positions. The terminal phalanges are spatulate and the skin has a soft, doughy texture with some hyperextensibility.

Affected children have a characteristic oval face, with a long upper lip, protuberant eyes, and sometimes mild scleral blueness (fig 1)

Clinical manifestations of SEMDFL

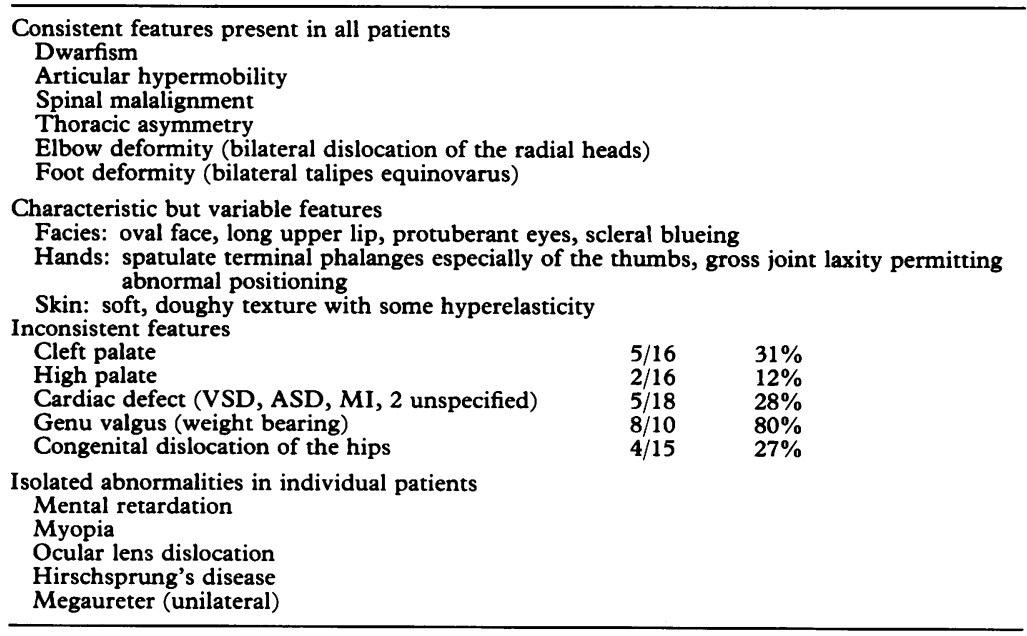

These facial features are variable and it must be emphasised that the appearance is not outside the normal range; nevertheless ostensibly unrelated children with the condition tend to have a closer resemblance to each other than to their unaffected sibs. The typical facies tends to become less obvious in later childhood.

Cleft palate is present in about $30 \%$ of affected children and a structural cardiac defect (VSD, ASD, MI) occurs in a similar proportion. Isolated abnormalities which have been encountered in individual patients include mental retardation, myopia, ocular lens dislocation, Hirschsprung's disease, and unilateral megaureter. It is uncertain whether these abnormalities are genuine inconsistent syndromic components or merely chance concomitants.

Walking may be delayed because of articular laxity but the majority of children eventually become ambulant. The knees are unstable and genu valgus on weight bearing is very frequent (fig 2). Other secondary manifestations of joint laxity become apparent at this stage, notably dynamic pes planus and hallux valgus. Spinal malalignment progresses during the first decade and the majority of affected children develop a severe kyphoscoliosis (fig 3). The clinical manifestations of SEMDJL are summarised in the table.

\section{Natural history}

The tissues are not fragile and healing is unimpaired. For these reasons, operative repair of palatal clefts and cardiac abnormalities does not pose any special problems.

The combination of ligamentous laxity and structural abnormalities of the vertebral bodies renders malalignment of the spine very refractory to treatment. External bracing is usually ineffective and some kind of internal fixation is often necessary. Despite these measures, progression is usual and a significant proportion of affected children develop spinal cord compression and paraplegia. Cardiorespiratory embarrassment owing to the malaligned dorsal spine and deformed thorax, plus a possible structural cardiac defect, all contribute to an unfavourable prognosis. In the author's experience, 10 affected children have died in the first or second decade and so far only two have reached adulthood. Equally, no affected persons over the age of 25 years have been encountered, despite more than two decades of investigation of skeletal dysplasia syndromes in the populations of South Africa.

\section{Radiological manifestations}

Radiographic abnormalities are present throughout the skeleton and limited skeletal survey permits the establishment of a firm diagnosis.

Changes in the vertebral bodies, epiphyses, and metaphyses are indicative of a disorder in the spondyloepimetaphyseal dysplasia category. During infancy the vertebrae are ovoid and in later childhood they become flattened and misshapen, with irregular endplates and 


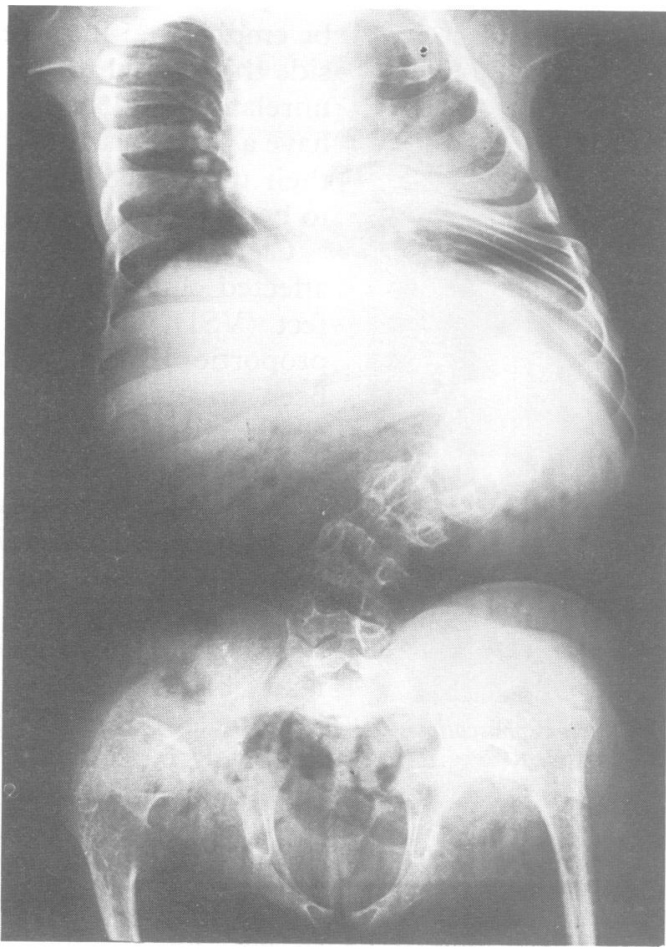

Figure 4 Anteroposterior view of the spine of an adolescent with SEMDFL. The spine shows gross malalignment and the vertebral bodies are very dysplastic. (From Beighton and Kozlowski. Skeletal Radiol 1980; 5: 205-12.)

some narrowing of the vertebral canals. Spinal malalignment is universal and often severe (fig 4). In the pelvis the iliac wings are large and flared, while the iliac bodies are hypoplastic and the pubic bones are narrow. Development of the femoral capital epiphyses is retarded and some degree of subluxation of the hip is usual. At a later stage irregular ossifica-

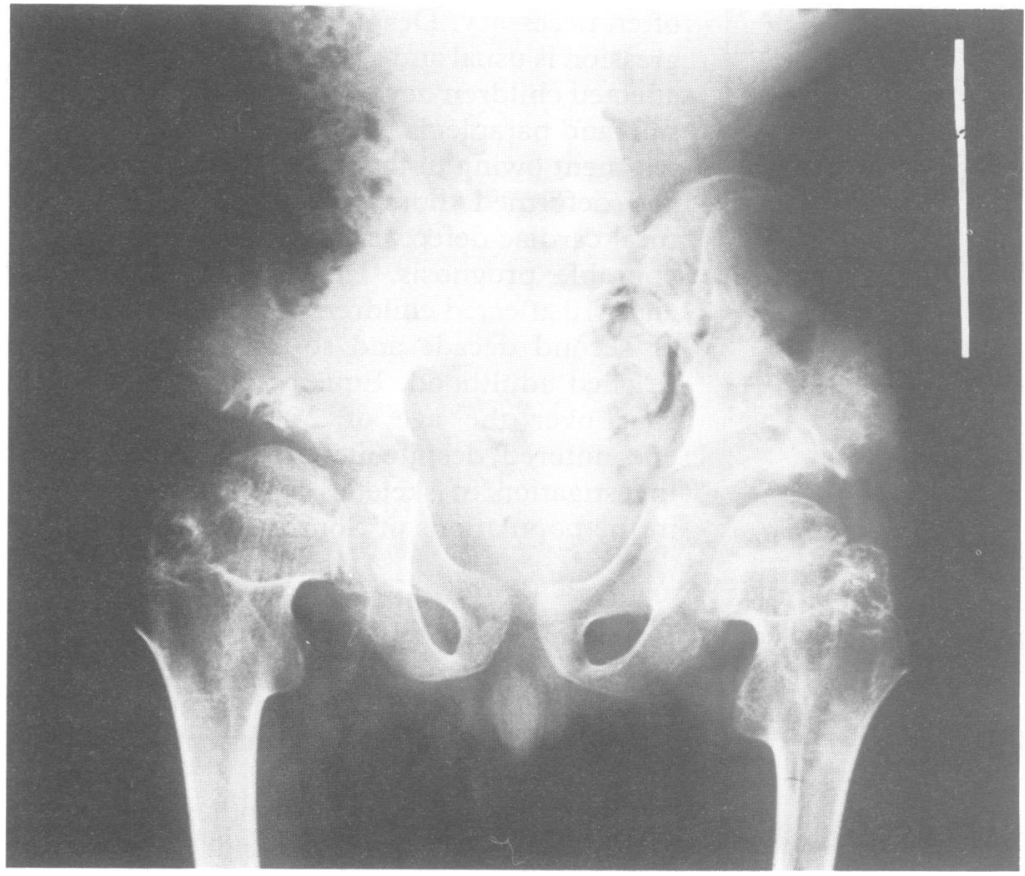

Figure 5 Anteroposterior view of the pelvis of an affected girl aged 8 years. The pelvis is small and the acetabula are irregular. The femoral necks are short and the trabecular pattern of the metaphyseal regions and upper portions of the femoral shafts are very abnormal. Lucent cysts are evident in the upper femora. (From Beighton and Kozlowski. Skeletal Radiol 1980; 5: 205-12.)

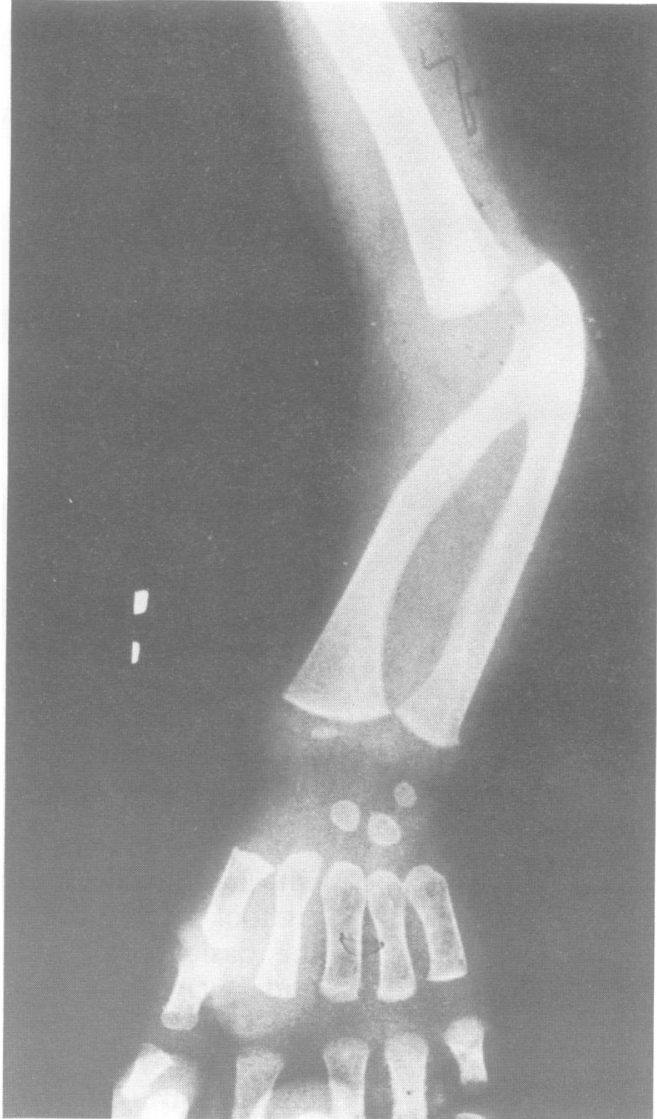

Figure 6 Anteroposterior view of the forearm of an affected child. The radial head is subluxed and the radial shaft shows the characteristic bowing. (From Beighton and Kozlowski. Skeletal Radiol 1980; 5: 205-12.)

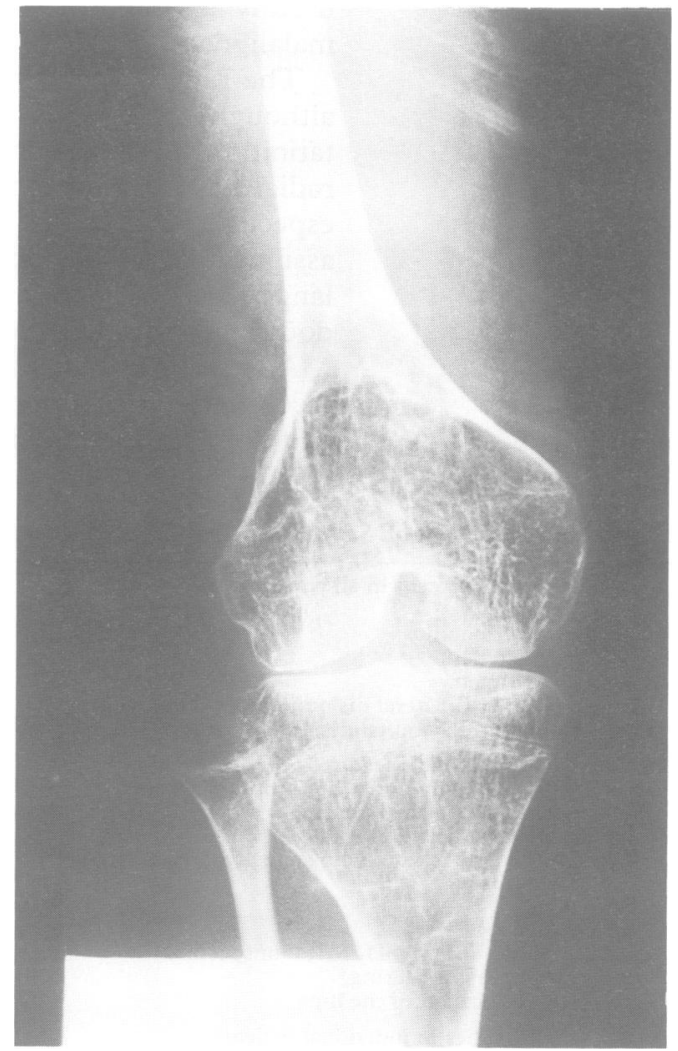

Figure 7 Anteroposterior view of the knee of an affected girl aged 11 years. The ends of the long bones are expanded and osteoporotic, with very abnormal trabeculation. (From Beighton and Kozlowski. Skeletal Radiol 1980; 5: 205-12.) 


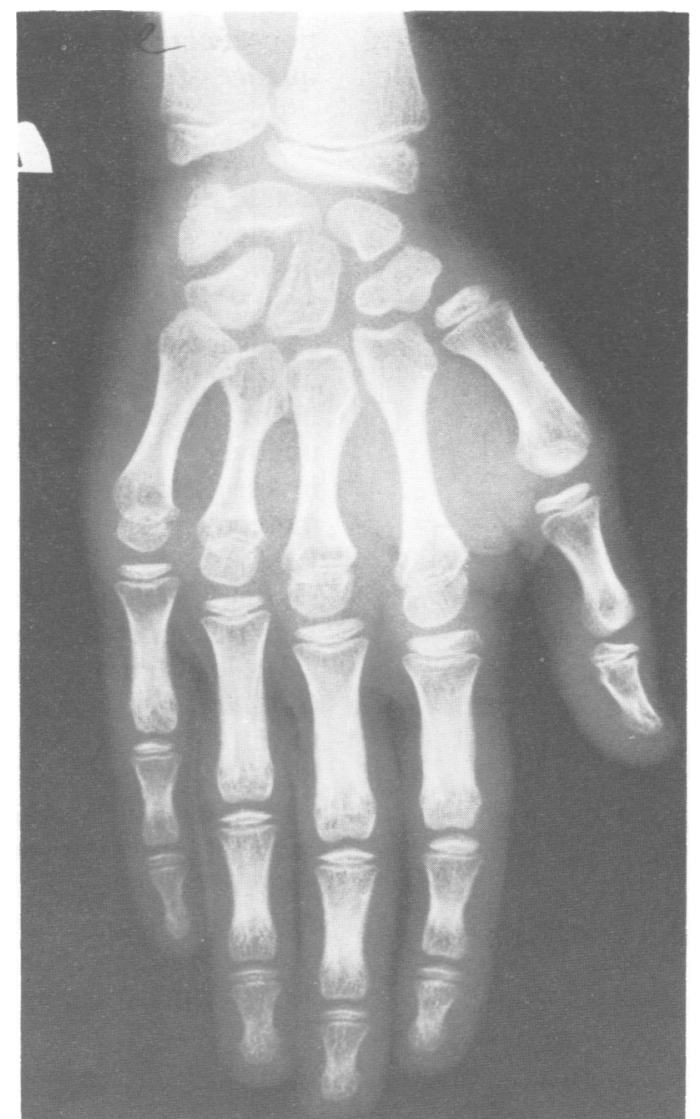

Figure 8 Anteroposterior view of the hand and wrist of the girl depicted in fig 2 at the age of 10 years. The tubular bones show minor epimetaphyseal changes and the shafts are shortened, especially in the metacarpals. The lunate and triquetral bones are fused. (From Beighton and Kozlowski. Skeletal Radiol 1980; 5 205-12.

tion of the acetabular cavities is evident, with small femoral capital epiphyses and coxa valga deformity (fig 5). In the arms, the distal end of the humerus is wide and somewhat irregular. The proximal portion of the ulna is bulky and the distal region is shortened. The radius is characteristically short and bowed and the radial head is dislocated (fig 6). All long bones are shortened and slender, with metaphyseal widening. The trabecular pattern is abnormal

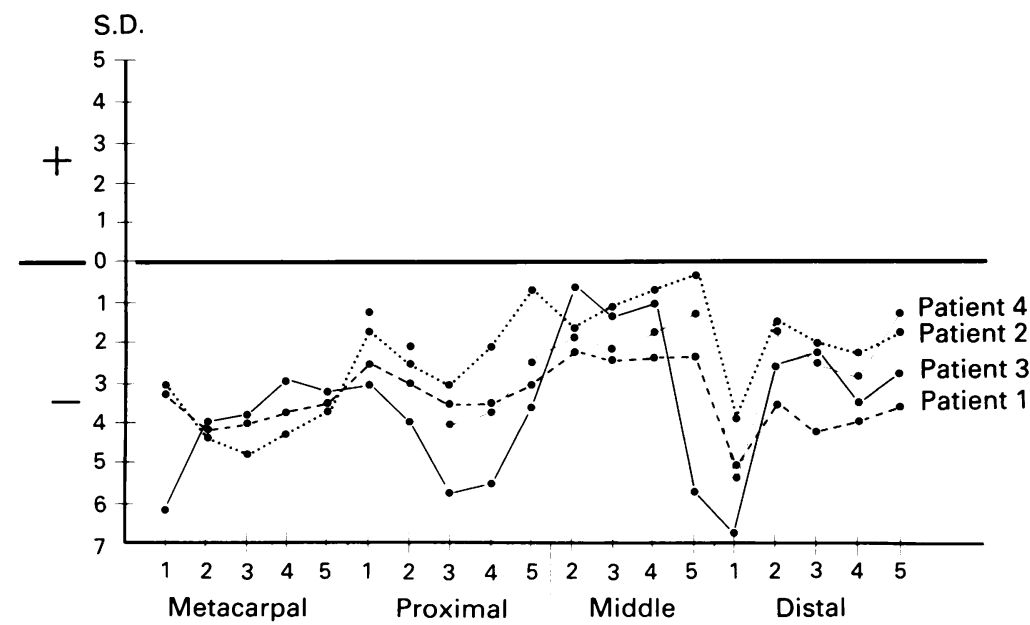

Figure 9 Pattern profile analysis which shows that four affected persons all have similar shortening of the tubular bones of their hands. (From Beighton and Kozlowski. Skeletal Radiol 1980; 5: 205-12.) throughout the limb bones; this feature is progressive and in the late stages cystic changes develop in the metaphyses and juxtametaphyseal regions (fig 7). The shafts of the tubular bones of the hands are shortened (fig 8). Pattern profile analysis by the method of Poznanski et $a l^{\beta}$ shows that these changes are maximal in the metacarpals and terminal phalanges (fig 9).

\section{Differential diagnosis}

In the newborn, the short limbed dwarfism, generalised skeletal dysplasia, and deformities of the elbows and feet are suggestive of diastrophic dysplasia. Moreover, the characteristic facial appearance in SEMDJL resembles that of diastrophic dysplasia, and the possible presence of a cleft palate further suggests this condition.

In view of anatomical distribution of the radiological abnormality, an initial misdiagnosis of non-specific spondyloepiphyseal dysplasia, or spondyloepimetaphyseal dysplasia, is not unusual.

The lax joints, spatulate fingers, and dwarfism may suggest the Larsen syndrome, while the articular hypermobility plus the skin hyperelasticity, if pronounced, may lead to confusion with the Ehlers-Danlos syndrome.

In the author's experience, all the above mentioned misdiagnoses have been applied to affected children. Recognition of the typical radiographic changes, however, permits diagnostic accuracy. In particular, the characteristic radial bowing is a useful sign during infancy, while in later childhood the severity of the progressive kyphoscoliosis sets SEMDJL aside from other similar disorders.

\section{Genetics}

Of the first 18 affected children ascertained by the author in southern Africa, eight were boys and 10 were girls. These persons were members of 13 nuclear families, of which four were known to be consanguineous, and the parents were unaffected in every instance. Three of the families had common progenitors within the last four generations (fig 10), while others were shown to share more remote antecedants (see below). On this basis, autosomal recessive inheritance is probable. Obligate heterozygotes are normal in every way, and they do not display any minor phenotypical abnormalities.

The affected families were all members of the Afrikaans speaking community of southern Africa, but it is noteworthy that several had Germanic surnames, while others lived in the eastern region of the Cape Province, which received substantial German immigration during the middle of the last century. A high proportion of these immigrants had fought with the German Legion in the British army during the Crimean War of 1854-1855. After peace was declared, political issues precluded these German soldiers from returning to their homeland. They were therefore offered the option of being integrated into the British forces which were departing for India for the 


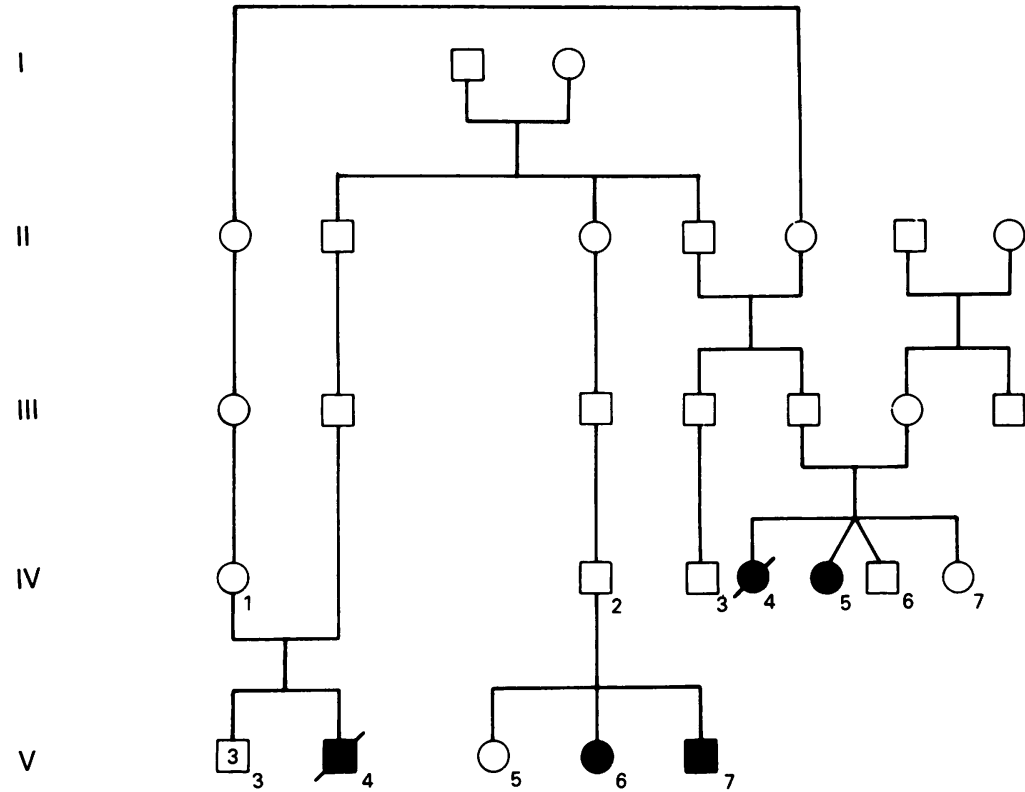

Figure 10 Pedigree showing three affected families with common antecedents. (From Beighton et al. Clin Genet 1984; 26: 308-17.)

suppression of the Mutiny of 1857, or of resettlement in the Eastern Cape. Many chose this latter option, and Germanic place names and family names are still common in this region. It initially seemed plausible that the SEMDJL gene might have reached South Africa by this route, but comprehensive genealogical investigation of eight of the affected families subsequently showed that they all had ancestral links with two females who were 12 or more generations removed from the persons with SEMDJL. ${ }^{7}$ These women had multiple marriages and cohabitations during the late 17 th and early 18 th centuries. Their ancestors lived in the Holy Roman Empire, which included most of contemporary Germany, Holland, Belgium, and Prussia but the precise origins of the SEMDJL gene cannot be further identified.

Apart from the persons with SEMDJL in South Africa, the author has been informed of affected persons in France, North America (Asiatic stock), and Germany (Turkish and German stock) and it is evident that the gene is rare but widespread. The comparatively high gene frequency in a subgroup of the popula- tion of southern Africa is probably the consequence of the founder effect.

\section{Basic defect}

In view of the recent or remote ancestral links between several of the affected families in South Africa, it can be concluded that the condition is genetically homogeneous in this country. DNA has been banked from a significant proportion of affected persons and their unaffected first degree relatives, and linkage investigations have been undertaken in the molecular laboratory of the MRC Research Unit for Medical Genetics, Department of Human Genetics, University of Cape Town. Significant negative results have been obtained with candidate loci, including COL1Al, COL1A2, COL2A1, fibrillin, elastin, and COL10A1. Another possible candidate is the protein decorin, which may be involved in joint laxity syndromes such as SEMDJL. In view of the autosomal recessive nature of SEMDJL, it is possible that the defect lies in the processing, rather than the primary structure of collagen, and elucidation of the molecular pathogenesis may therefore be difficult.

I am grateful to Gillian Shapley for typing the manuscript with her usual efficiency. This work was supported by grants from the Medical Research Council of South Africa, the Harry Crossley Foundation, the Mauerberger Foundation, and the University of Cape Town Staff Research Fund.

1 McKusick VA. Mendelian inheritance in man 10th ed. Baltimore: Johns Hopkins University Press, 1992.

2 Spranger J. International classification of osteochondrodysplasias. The International Working Group on Constitutional Diseases of Bone. Eur f Pediatr 1992;151:407-15.

3 Beighton P, Kozlowski K. Spondylo-epi-metaphyseal dysplasia with joint laxity and severe, progressive kyphoscoliosis. Skeletal Radiol 1980;5:205-12.

4 Beighton P, Kozlowski K, Gericke G, Wallis G, Grobler L. Spondylo-epi-metaphyseal dysplasia with joint laxity and severe, progressive kyphoscoliosis. S Afr Med $\mathfrak{f}$ 1983;64:772-6.

5 Kozlowski K, Beighton P. Radiographic features of spondylo-epi-metaphyseal dysplasia with joint laxity and progressive kyphoscoliosis. Review of 19 cases. ROFO 1984;141:337-41

6 Beighton P, Gericke G, Kozlowski K, Grobler L. The manifestations and natural history of spondylo-epi-metaphyseal dysplasia with joint laxity. Clin Genet 1984;26:308-17.

7 Torrington $M$, Beighton $P$. The ancestry of spondyloepimetaphyseal dysplasia with joint laxity (SEMDJL) in South Africa. Clin Genet 1991;39:210-13.

8 Poznanski AK, Garn SM, Nagy JM, Gall JC. Metacarpophalangeal pattern profiles in the evaluation of skeletal malformations. Radiology 1972;104:104-11. 